\title{
Nursing Nefrologico e coincidenze casuali
}

Sono grata a Sandro per la sua proposta e mi piace pensare che nulla accada "per caso". Quella casualità scelta dalla scienza per meglio descrivere la variabilità del reale e del vero. Inoltre, la conoscenza storica dà la possibilità di meglio collocare, valutare e reagire alla variabilità degli eventi quotidiani.

Casualità: la serie di articoli proposti compare proprio ora che stiamo discutendo di competenze infermieristiche avanzate e, direi anche, contrastate da un pensiero storico "altro". II pensiero di coloro che faticano a riconoscere i cambiamenti che loro malgrado hanno concorso a creare: infermieri che temono la responsabilità connessa alla professionalità, medici che persistono nella considerazione ancillare dell'infermiere. La storia di Sandro potrà spiegare meglio perché oggi la gestione del paziente con malattia renale cronica (MRC), nei vari stadi di percorso di cura e assistenza, può e deve essere condivisa con infermieri che abbiano maturato competenza esperienziale e/o svolto percorsi formativi, nonché collocati in posizioni contrattuali congrue.

Storia: della storia iniziale vorrei salvare il clima lavorativo, dove rispetto e reciprocità erano elementi essenziali per affrontare il nuovo della tecnologia e lo sconosciuto della cura. Anche oggi abbiamo sfide da affrontare: i nostri pazienti sono sempre più fragili e comorbidi, quelli giovani sono spesso stranieri con usi, costumi e intendimenti diversi. Le sfide dell'ignoto ci sono ancora, anzi più che mai; sarebbe molto meglio per tutti noi - pazienti, infermieri e nefrologi - se ci impegnassimo a creare sinergie operative, piuttosto che programmare strategie di assolutismo e rivalsa.

II Nursing Nefrologico è iniziato con competenze tecniche nell'ambito di una forma di medicina innovativa. Con il tempo le conoscenze sono state acquisite, rigenerate e adeguate ai bisogni delle persone. Ritengo che il ruolo infermieristico nel tempo abbia concorso al benessere e al prolungamento della vita dei pazienti, tanto quanto la specialità nefrologica ha concorso a creare tecnologie e farmaci per curare la malattia.

Auspico che il meglio della storia che ha permesso di rendere il trattamento dialitico disponibile per tutti, si riproponga oggi in una gestione più lungimirante, articolata e personalizzata della MRC, a salvaguardia della vita, della persona e del nostro Sistema Sanitario Nazionale.

Il Nursing Nefrologico in Italia è stato caratterizzato da una vision associativa nazionale e internazionale, di cui Sandro è stato una delle pietre miliari. Ringrazio lui e tutti i compagni di avventura per aver creato la Filiale Italiana Edtna/Erca e per aver concorso a diffondere conoscenze e creare consapevolezze. Gli articoli di Sandro saranno certamente fonte di ulteriore ispirazione e fertile terreno di confronto tra professionisti e professioni.

Marisa Pegoraro

Presidente Filiale Italiana EDTNA/Erca 